\title{
Postsäkulares Placemaking im oberpfälzischen Konnersreuth: Sakralisierung, Kulturerbe, Eigensinn
}

\author{
Thomas P. Funk \\ Institut für Europäische Ethnologie, Humboldt-University Berlin, Berlin, Germany \\ Correspondence to: Thomas P. Funk (thomas_p_funk@gmx.de)
}

Received: 27 September 2016 - Revised: 10 May 2017 - Accepted: 29 May 2017 - Published: 18 July 2017

\begin{abstract}
Kurzfassung. The initiation of the beatification process of catholic stigmatist Therese Neumann of Konnersreuth/Bavaria (1898-1962) in February 2005 marks the beginning of the touristic marketing of her birth place in the context of EU-regionalization. In the case concerned, I argue, the Postsecular emerges in the evolution of new forms of EU-regional governance and in the controversial valorisation of a catholic popular cult as "cultural heritage". I examine postsecular placemaking in Konnersreuth as a process, in which actors of the local civil society, the official church and the EU bureaucracy stage Therese Neumann by the valorisation of her material heritage, by sacralization and profanation. In doing this, actors renegotiate boundaries and transitions between the religious and the secular sphere, as well as between the regional, the global and the singular universality of grace. In their productions of space, local actors mediate between tourist and spiritual experiences of authenticity, between the popular demand for "heritage" and the awaiting for the religious event of grace. The postsecular, I argue, emerges in the insistence of parts of the local civil society to authorize the deceased Therese Neumann herself as an - though unwilling - supranatural actor in the placemaking process and in their legitimation of their own (un-)willingness to participate in the sacralization and touristification of "their" heritage with the will of the stigmatist. The article shows, how religious belief can play the role of a ressource as well as a disruptive factor in postsecular placemaking processes and neoliberal "governing by community".
\end{abstract}

\section{Einleitung}

Konnersreuth in der nördlichen Oberpfalz, ein Ort mit etwa 1800 EinwohnerInnen, der durch die bayerische Stigmatisierte und Seherin Therese Neumann (1898-1962) Ende der 1920er Jahre in aller Welt bekannt wurde, wird gegenwärtig im Rahmen der EU-Regionalisierung als Kulturerbe inwertgesetzt. Seit ihrer erstmaligen Stigmatisierung 1926 bis zu ihrem Tod zogen Tausende BesucherInnen in die entlegene Ortschaft nahe der bayerisch-tschechischen Grenze, um dort ZeugInnen zu werden, wie Therese Neumann die blutige Passion Jesu Christi - sein Leiden und seinen Tod am Kreuz - mitvollzog. Regelmäßig freitags - im Laufe ihres Lebens insgesamt 600 Mal (Panzer, 1991:52) - habe sie, in ihrem Bett im elterlichen Wohnhaus liegend, in Visionen ekstatisch entrückt, das Leiden des Religionsstifters körperlich nachvollzogen und in Mimik und Gestik für die BetrachterInnen nachvollziehbar gemacht. Das Aufbrechen und Bluten ihrer Stigmata - der Wundmale Jesu Christi an ihrem Körper - begleitete ihren stets mehrstündigen Mitvollzug der Passion Christi. Therese Neumann war und ist umstritten. Ihr Freundeskreis, der „Konnersreuther Kreis“ - katholische Bischöfe, Theologen, Adlige, monarchistische Großgrundbesitzer und Publizisten aus dem süddeutschen Raum - widmete sich der Verteidigung der Stigmatisierten gegen Hysterie- und Betrugsvorwürfe und deutete Therese als Sühneseele (Kane, 2002), als Zeichen der göttlichen Präsenz und als Warnung vor der menschlichen Hybris in der säkularen Moderne. Als einfacher Frau aus dem Volke sei Therese die Gnade zuteil geworden, die göttliche Wahrheit zu erkennen, die der säkularen Wissenschaft und Politik verschlossen bleibe (Steiner, 1985:197). Mehr als vierzig Jahre nach ihrem Tod verkündete der Regensburger Bischof Gerhard Ludwig Müller am 13. Februar 2005 die Eröffnung des kirchlichen Seligsprechungsprozesses für Therese Neumann. Der Leiter der Abteilung für Selig- und Heiligsprechungsverfahren im Bistum Regensburg stellte vier grundlegende Elemente des ,geistli- 
chen Erbes" Therese Neumanns heraus: ihren Hinweis auf die stellvertretende Sühne Christi, die ewige Anbetung des „eucharistischen Herrn“, Thereses vorbildlichen Widerstand gegen ,widerchristliche und menschenverachtende Zeitströmungen“" wie das „gottlose Nazi-Regime“ und schließlich die Mission und Glaubensverbreitung (o. A. [Schwager, G.] 2005, 11f.).

Therese Neumann starb am 18. September 1962, knapp einen Monat vor der Eröffnung des Zweiten Vatikanischen Konzils. Das Aggiornamento des Konzils interpretiert José Casanova, dessen Beitrag die aktuelle Debatte um „öffentliche Religionen“ prägt, als endgültigen Abschied der katholischen Kirche vom klerikalen Antimodernismus und als Wende hin zum zivilgesellschaftlichen Engagement gegen Diktatur und für Demokratie (Casanova, 2009:46, 71f., 226). Die Eröffnung des Seligsprechungsprozesses fiel in eine Zeit der konservativen Restauration der katholischen Amtskirche durch Papst Benedikt XVI., der breiten Debatte um den zivilgesellschaftlichen Beitrag ,öffentlicher Religion“ in einer „,postsäkularen“ Gesellschaft und der Artikulation von Forderungen einer christlichen Fundierung EU-Europas. Etwa zeitgleich setzte die Inwertsetzung des physisch-materiellen Erbes Therese Neumanns als „regionale Besonderheit“ und „Alleinstellungsmerkmal“ Konnersreuths mithilfe von EUFörderprogrammen zur Entwicklung ländlicher Räume ein. Das Erbe Therese Neumanns wird nun Teil einer touristischen Raumproduktion: einer „Sakralen Landschaft“.

Ich untersuche am Placemaking in Konnersreuth, wie AkteurInnen der lokalen Zivilgesellschaft, der Amtskirche und der EU-Bürokratie das materiell-physische wie symbolische Erbe Therese Neumanns in Prozessen der Heritageifizierung, Sakralisierung und Profanierung inszenieren und dabei Grenzen und Übergänge zwischen dem Religiösen, Säkularen und Profanen wie zwischen regional Besonderem, Globalem und der singulären Universalität des religiösen Ereignisses der Gnade neu verhandeln. Ich stelle dar, wie die AkteurInnen in ihren Raumproduktionen (Low and Lawrence-Zuñiga, 1999) zwischen touristischen Erlebnissen des Authentischen und spirituellen Erfahrungen, zwischen der Nachfrage nach Kulturerbe und dem Hoffen auf religiöses Heil und Heilung vermitteln. Dem Unvernehmen (Rancière, 1999) und Eigensinn einer durchaus nicht immer einigen lokalen Zivilgesellschaft sowie den Präsenzeffekten des Heiligen unter postsäkularen Bedingungen gilt meine besondere Aufmerksamkeit. ${ }^{1}$

\footnotetext{
${ }^{1}$ Meine Feldstudie verstehe ich als epistemologische Befragung von Kategorien im Sinne der Diskursanalyse (Asad, 2003; Landwehr, 2008). Sie basiert auf ethnographischen Feldforschungen, die ich in den Jahren 2006 bis 2009 durchgeführt habe (Experten-)Interviews, teilnehmender Beobachtung, dichten Beschreibungen des materiell-physischen „Erbes“ sowie der Praktiken seiner Inszenierung und seines Gebrauchs - sowie der Analyse von Publikationen der am placemaking beteiligten AkteurInnen und Institutionen. GesprächspartnerInnen werden namentlich nicht genannt.
}

Das endogene Potential der ,Sakralen Landschaft“ in Konnersreuth sei kurz benannt: Das Wohn- und Geburtshaus Therese Neumanns („Reslhaus“) auf dem Marktplatz ist als Andachtsstätte geöffnet. Die barocke Pfarrkirche wird für die monatlichen Gebetstage um die Seligsprechung Therese Neumanns genutzt. Das „Reslgrab“ auf dem Friedhof mit der Votivkapelle ist das stille Zentrum der Verehrung. Zwei von Therese Neumann mitbegründete Klöster, „St. Joseph Fockenfeld“ und das Anbetungskloster „Theresianum“, gelten als ihr geistiges Vermächtnis. Der „Reslgarten“, die originalgetreue Rekonstruktion ihres Blumengartens, wurde 2006 eröffnet. Für das geplante „Dokumentations- und Begegnungszentrum Schafferhof“" mit „Reslmuseum“ waren die Fördergelder im Jahr 2009 weitgehend gesichert; nach einem Aufschub des Projekts wurden sie 2017 neu beantragt.

In meiner Analyse konzentriere ich mich auf drei aktuelle Raumproduktionen: „Reslhaus“, „Reslgarten“ und „Schafferhof". Anhand der Ausstellungsobjekte in der Andachtsstätte „Reslhaus“" verdeutliche ich Positionen amtskirchlicher Akteure im Seligsprechungsprozess, die das Leiden der Stigmatisierten als Mitwirkung an Jesu Christi Erlösungswerk das „Erbe“ sakralisieren und auf einer strikten Trennung der religiösen und säkularen Sphären beharren. Im „Reslgarten“ stellen AkteurInnen der Zivilgesellschaft postsäkulare Vermittlungen zwischen gouvernementalen und religiösen Konzeptionen von Gesundheit, Lebensqualität und Subjektivität her. In der Debatte um das geplante „Dokumentations- und Begegnungszentrum Schafferhof" wird deutlich, wie stark Kulturerbe-Strategien und Ansätze der regionalen Governance auf Konsens angewiesen sind und wie die Bezugnahme auf Therese Neumann als übernatürliche Akteurin im Placemaking-Prozess eine religiöse Ressource lokalen Eigensinns darstellt.

\section{Postsäkulares zwischen Kulturökonomie und Governing Community}

Als postsäkulares Placemaking bezeichne ich die physischmaterielle wie symbolische Inszenierung religiöser Präsenz sowie die Verflechtungen zwischen Lokalem und potentiell Globalem, die dabei hergestellt werden. Ich gehe davon aus, dass sich im postsäkularen Placemaking neuartige Verhältnisse des Politisch-Ökonomischen und Religiösen artikulieren.

Das „Postsäkulare“ wird gemeinhin als anhaltende Präsenz des Religiösen innerhalb säkularisierter Sozialstrukturen der Gegenwart verstanden, die die Gleichsetzung von Modernisierung mit Säkularisierung obsolet macht und Fragen nach den Möglichkeiten eines Zusammenlebens von Religiösen, Humanisten und Säkularisten in einer pluralen Gesellschaft stellt (Molendijk et al., 2010). Geographische Theorien des Postsäkularen nehmen meist religiös-politische Konstellationen im urbanen Raum europäischer Metropolen in den Blick, um öffentliche Religion als treibende Kraft 
in räumlichen, sozialen und politischen Transformationsprozessen darzustellen (ebd., Oosterbaan, 2014; Beaumont et al., 2011). Das Postsäkulare erscheint dabei als stets vorläufiges Resultat einer Auseinandersetzung mit der Diversität und kulturellen Pluralität urbanen Lebens (Gökarıksel and Secor, 2015). Fallstudien untersuchen die Kooption religiöser AkteurInnen in Governance-Prozessen ebenso wie des Widerstands dagegen, der sich im partnerschaftlichen Handeln religiöser und nicht-religiöser AkteurInnen äußern kann (Cloke and Beaumont, 2012). Einzelne Studien untersuchen die Rolle touristischer und religionstouristischer Raumpraktiken bei der Entstehung postsäkularer Verhältnisse (Nilsson, 2016). Im vorliegenden Fall zeigt sich die postsäkulare Präsenz im Modernisierungsprozess eines ländlichen Raums in der Produktion von Kulturerbe als Ressource von Ökonomie und regionaler Identität. Mit José Casanova gehe ich davon aus, dass sich das Verhältnis des Säkularen und Religiösen im Prozess der europäischen Integration ändert $(\mathrm{Ca}-$ sanova, 1994:9, 70; Casanova, 2009:24, 35, 87ff., vgl. Beiträge in Malik, 2010). ${ }^{2}$ Das Postsäkulare, so meine These, entsteht im Rahmen der anhaltenden postfordistischen Transformation des Akkumulationsregimes (Heeg, 2001): im Wandel von staatlichem Government zur regionalen Governance, in zunehmend heterogenen Arrangements der ,EURegionalisierung“ (Johler, 2005) und in der umstrittenen Inwertsetzung religiöser Kultur in ökonomischen Innovationsprozessen

Kulturerbe verstehe ich als metakulturelle Produktion (Kirshenblatt-Gimblett, 2004), die Orte, Objekte und Praktiken in neue Bedeutungskontexte versetzt, symbolisch und ökonomisch aufwertet und Gemeinschaft darüber neu identifiziert (Tauschek, 2013). Ausstellbarkeit und Besuchbarkeit (Tschofen, 2007) stellen gemeinsame Merkmale des Kulturerbes und religiöser Sakramentalien, Heiliger Stätten und Wallfahrtsorte dar. Die Produktion von Kulturerbe wird von der EU im Rahmen der Entwicklung einer europäischen Identität aktiv vorangetrieben (Shore, 2000) und stellt als Teil der postfordistischen Kulturökonomie einen bedeutenden Wirtschaftsfaktor dar. Die Popularität von Fragen des Erbes und der Erinnerungskultur führt die Kulturanthropo-

\footnotetext{
${ }^{2}$ Casanova unterscheidet verschiedene Modelle der Trennung von Säkularem und Religiösem und der staatlichen Regulierung von Religion (Casanova, 2009:93). Einem angelsächsischprotestantischen Pfad der Säkularisierung als Durchdringung religiöser und säkularer Sphären stellt er einen französischen, lateinischkatholischen Pfad der Laizisierung gegenüber, die durch eine zivilkirchliche und laikal-klerikale antagonistische Dynamik gekennzeichnet sei (Casanova, 2009:87f.). „Hier werden die Grenzen zwischen dem Religiösen und dem Säkularen rigide aufrecht erhalten [... ].“ (ebd.: 88) Deutschland, ein protestantisches Land mit starker katholischer Minderheit, entwickelte sich im ausgehenden 19. Jahrhundert zum Bollwerk eines liberalen katholischen Ultramontanismus (Casanova, 2009:35). Das isolierte „katholische Milieu“ ist seit den 1960er Jahren in Auflösung begriffen.
}

login Sharon Macdonald dazu, Europa als Memoryland zu bezeichnen (Macdonald, 2013).

Placemaking verstehe ich in Anlehnung an Doreen Massey (1994) als umstrittene Verknüpfung lokaler und potentiell globaler Prozesse - in diesem Fall der Seligsprechung der katholischen Kirche und der EU-Europäisierung - zu einer Assemblage, die Geschichtskonstruktionen und $\mathrm{Zu}$ kunftsentwürfe verbindet (Massey, 1995). Diese Assemblage kann, den Vorstellungen religiöser Akteure gemäß, auch nicht-menschliche Aktanten wie Götter, Geister und Heilige umfassen (Orsi, 2010). ${ }^{3}$

Das postsäkulare touristische Placemaking profitiert von der Ausstellbarkeit des (subalternen, peripheren, ländlichen, etc.) Anderen: Nie man selbst, immer der Andere lebt Formen unmittelbarer Religiosität, überspitzt Slavoj Žižek (2001:7-10). Er beschreibt die Kulturalisierung von Religion als ,,postsäkulare“ Option“ im gegenwärtig gültigen „Rahmen des suspendierten Glaubens“ (Žižek, 2003:10). Allen öffentlichen Bezugnahmen auf Religion sei eine individuelle Distanzierung vom unmittelbaren, naiven Glauben einbeschrieben, die sich etwa in der Respektbezeugung vor Religionen als „kulturellem Erbe“ des eigenen Landes oder den ,kulturellen Schätzen“ der gesamten Menschheit äußere (Žižek, 2003:8-10). Žižek pointiert: „,Kultur“ ist der Name für all jene Dinge, die wir tun, ohne wirklich an sie zu glauben, ohne sie ,ernstzunehmen“."(Žižek, 2003:9).

Die Gegenwart scheint nicht nur vom Kulturerbe, sondern auch vom Thema Religion ,,besessen“ (Macdonald, 2013:1; Casanova, 2011:76) und anhaltend damit beschäftigt. Die Rede von der Rückkehr „öffentlicher Religion“ in Arenen moralischer und politischer Auseinandersetzung ist inzwischen Gemeinplatz. José Casanova und Jürgen Habermas, die profiliertesten liberalen Theoretiker des Postsäkularen, verstehen Religion dabei weniger als Herrschaftsverhältnis denn als Wissensressource und beschreiben das Postsäkulare als Veränderung im kulturellen Selbstverständnis des säkularen Europa, das Moderne und Säkularität entkoppelt und „Möglichkeiten der Transzendenz in unserem säkularen Rahmen der Immanenz“ zulässt (Casanova, 2011:78). „Öffentliche Religion“ begrüßt Casanova als Motor gesellschaftlichen Wandels und einer ethischen Kritik an der Autonomie der ökonomischen und staatlichen Sphäre, die aber - dies setzt er implizit voraus - die kapitalistische Ökonomie und den Nationalstaat nicht grundsätzlich infrage stellt. Casanova trägt normative Ansprüche an öffentliche Religionen heran: Beiträge zur zivilgesellschaftlichen Selbstverständigung seien legitim, sofern sie kompatibel mit den differenzierten Strukturen der Moderne seien und transzendente (heißt dies: irrationale?) Wahrheitsansprüche aus dem öffentlichen Austausch rationaler Argumente ausklammerten (Casanova, 1994:7f., 223, 2009:108ff.). Casanova hält es für vorstellbar,

\footnotetext{
${ }^{3}$ Damit vertrete ich einen Religionsbegriff, der die Herstellungsprozesse und die Wahrheits- und Realitätseffekte religiöser Materialität und Medialität in den Blick nimmt (Meyer, 2014).
} 
dass , ,[... ] after all the beatings it had received from modernity, religion could somehow unintentionally help modernity save itself." (Casanova, 1994:234) Sein Plädoyer für religiöse Beiträge zur Selbstverständigung der Zivilgesellschaft lässt die staatliche Aktivierung religiöser Gemeinschaften und Individuen sowie die gouvernementale Aneignung religiöser Sinnstiftung als Legitimationsressource in Zeiten der Krise empfehlenswert erscheinen.

\section{EU-Regionalisierung: Produktion von Kulturerbe, Identität und Konsens}

Neben die liberale Inanspruchnahme von Religion als ethischer Ressource einer säkular scheinbar anders nicht zu habenden „moralischen Sensibilität“ (Habermas) tritt eine kommunitaristische Stärkung religiöser Gemeinschaft als Hort gesellschaftlicher Werte, gegenseitigen Vertrauens, der Privatinitiative und Eigenverantwortung. Im Rahmen eines Governing by Community (Rose, 1999) werden religiöse Gemeinschaften und Organisationen - unter Ausklammerung ihrer religiösen Selbstdeutungen - in staatlichen Regierungsprozessen kooptiert (MetroZones, 2011). Über wirtschaftsgeographische Theoriebildung etwa der kalifornischen Schule findet der Gemeinschaftsdiskurs auch Eingang in die Praxis der Regionalentwicklung, der Entwicklung regionaler Netzwerke und kreativer Milieus (Rose, 1999:168).

Im Prozess der „EU-Regionalisierung“, in dem landwirtschaftlich geprägte ländliche Regionen als „Landschaft“ kulturalisiert und in Kategorien des Kulturerbes konzipiert werden (Johler, 2005), wird auch Religion adressiert. Seit dem Vertrag von Maastricht 1992 verschreibt sich die EU dem Schutz und der Förderung einer kulturellen „Einheit in Vielfalt" und begreift das kulturelle, religiöse und humanistische Erbe Europas als Quelle und Garantie universeller Werte wie Freiheit, Demokratie, Gleichheit und Rechtsstaatlichkeit (Shore, 2001). Religion und religiöse Gemeinschaften werden in der Folge zum Gegenstand von Förderpolitiken (vgl. die Beiträge in Malik, 2010).

Bayern eignet sich den Diskurs um die kulturelle Identität Europas auf spezifische Weise an: Josef Miller, von 1998 bis 2008 bayerischer Staatsminister für Ernährung, Landwirtschaft und Forsten, begreift die geschichtliche und kulturelle Identität Bayerns, die christliche Prägung und besonders die katholische Tradition seines Landes als fundamentalen „,bayerischen Beitrag zu einer künftigen Kultur Europas“ (vgl. Miller, 2004:6). Er beklagt die Beliebigkeit des anything goes, die Toleranz mit Gleichgültigkeit verwechsle, und fordert dazu auf, „Werthaltungen auf[zu]bauen, die in der Geschichte, in der Kulturgeschichte unseres Landes wurzeln" und die der ganzen Gesellschaft „,helfen, an einem Sinn der Geschichte und an der Bedeutung eines bayerischen Beitrags zu einer künftigen Kultur Europas festzuhalten.“(ebd.).
Konnersreuth (Landkreis Tirschenreuth) zählt zur bayerischen Planungsregion Nördliche Oberpfalz, die laut Regionalplan den allgemeinen Entwicklungsfortschritt noch nicht voll erreicht hat und deren Struktur zur Verbesserung der Lebens- und Arbeitsbedingungen daher nachhaltig gestärkt werden soll (BStMLF, 2001:12, 29). Die Inwertsetzung religiösen Kulturerbes mit EU-Förderprogrammen bildet einen Beitrag zum Modernisierungsziel, Lebensqualität zu steigern und den Tourismus zu befördern. Die Gemeinschaftsinitiative LEADER (frz. „Liaison entre actions de développement rural“) nimmt dafür ,kleine, homogene, sozial in sich geschlossene Gebiete mit gemeinsamer Tradition oder lokaler Identität" zum Ansatzpunkt für gebietsbezogene Entwicklungsstrategien (EU-Comm. 2000/C 139/05), die sich mit einem für die Identität des Gebiets typischen Schwerpunktthema auseinandersetzen. Die Regionalentwicklung mit LEADER ist mit Susanne Heeg eine „starke Wettbewerbsstrategie“, die eine Optimierung regionaler „Qualitätsbedingungen" durch Inwertsetzung regionaler Vermögenswerte und endogener Potentiale sowie die gezielte Stimulation gesellschaftlicher Aktivitäten (,Bürgerbeteiligung“) anstrebt, dabei soziokulturelle Faktoren wie Vertrauen, Kooperationsfähigkeit und Vernetzung stärkt und neue kollektive Vorstellungen und räumliche Leitbilder herstellt (Heeg, 2001:91, 98101).

1998 wurde im Landkreis Tirschenreuth die Lokale LEADER-Aktionsgruppe (LAG) InitiAKTIVkreis Tirschenreuth e. V. als Verein für das Regionalmarketing gegründet, an dem neben Repräsentanten der Gebietskörperschaften auch Vertreter der Wirtschafts- und Sozialverbände, Mitglieder von Vereinen und Privatpersonen aus dem Landkreis beteiligt sind. Im Jahr 2001 wurde ein Regionalmanagement am Landratsamt angesiedelt, welches in enger Zusammenarbeit mit der LEADER-Gruppe ,die in der Region vorhandenen Ideen, Akteure, Planungen und Projekte“ zusammenführt und in Prozessen der Bürgerbeteiligung eine „,konsensfähige" Strategie der Regionalentwicklung erarbeitet (LAG, 2000:88f.).

Für den Zeitraum 2000 bis 2006 erstellte die LEADERGruppe des Landkreises erstmals eigenständig ein Regionales Entwicklungskonzept (REK: LAG, 2000). In mehreren Workshops erfassten die Teilnehmer ,[...] die Vermögenswerte des Gebiets (Menschen, Aktivitäten, Landschaften, Erbe, Know-how) [...] durch Auflistung der einzigartigen Merkmale, die entwickelt werden können" (EU Commission, 2006:17). Dieses Verfahren, das eine Voraussetzung zur Möglichkeit der Einwerbung von Projektfördergeldern von der EU darstellt, verfolgt das Ziel einer „Bewusstseinsschärfung " (ebd.) der Akteure: einer gouvernementalen Transformation des Verhältnisses der Subjekte zu sich selbst und ihrer Region, die, nun als Erbe begriffen, Verpflichtungen artikuliert und Identifikation einfordert. Die „Aktivierung der Selbsthilfekräfte ländlicher Regionen“ (BStMLF, 2001:49) durch LEADER formuliert ein therapeutisches Programm, das eine Region fest mit Bevölkerung und Geschichte iden- 
tifiziert und als lebendigen Organismus begreift. Im REK drückt sich dieses therapeutische Programm im Leitbild ,Tirschenreuth - gesunder Wirtschaftsraum am Mittelpunkt Europas" aus (LAG, 2000:76). Gesundheit bedeutet dabei in einem ganzheitlichen Sinne „nicht nur physische, d. h. körperliche Gesundheit, sondern auch seelische, geistige und wirtschaftliche Gesundheit. Die Voraussetzung hierfür ist eine gesunde Region, die durch nachhaltige Strukturen und Prozesse, durch eine, gesunde ' regionale Identität, und durch ein wachsendes Miteinander geprägt ist.“ (LAG, 2000:76). Die Herstellung eines homöostatischen Gleichgewichts der Region verfolgt Wachstumsziele in einem ungleichen Wettbewerb der Regionen. Die Kräfte der „Globalisierung“ werden im Leitbild der Region externalisiert; dass grundsätzliche ökonomische und politische Fragen nicht auf regionaler Ebene lösbar sein könnten, wird dabei ausgeblendet.

Das Regionale Entwicklungskonzept 2007-2013 strebt die Inwertsetzung von Kulturerbe an. Der dafür eigens geprägte Begriff "Sakrale Landschaft" umreißt ein heterogenes Ensemble „regionaler Besonderheiten“: Klosterkultur, Krippenkunst, barocke Kirchen, Feldkreuze und Flurdenkmäler - und das „Projekt Konnersreuther Resl“ mitsamt Einrichtung eines Tourismusmanagements (LAG, 2007:80). „Sakrale Landschaft", so das Regionalmanagement, stehe für regionale Authentizität und Lebensqualität und stelle die Basis zur Generierung touristischen und wirtschaftlichen Mehrwerts dar (Interview am 21. Juli 2009). Die Lokale Entwicklungsstrategie (LES) 2014-2020 führt - unter dem Motto „Wir sind wandelbar“ - die Entwicklungsstrategie der Vorjahre und deren „Handlungsziel“ der Stärkung „regionaler Besonderheiten" fort (LAG, 2014:8, 12, 37). Bezüglich des „Entwicklungsziels 1“ der Stärkung von Tourismus, Kultur und Freizeitangeboten, das diesem Handlungsziel zugeordnet wird, heißt es: „Als innovative Fortsetzung und Weiterentwicklung unseres Themas ,Sakrale Landschaften ' aus dem REK 2007-2013 soll hier bei allen Aktivitäten der Bereich ,Gesundheit für Körper, Geist und Seele " eine besondere Rolle spielen.“ (ebd. 37) Damit werden religiöse und säkulare Diskurse um Gesundheit und Heilung in einen engen Zusammenhang gebracht.

Religiöse Kultur findet laut Regionalmanagement einen „breiten Rückhalt in der Bevölkerung“ des Landkreises. Sie sei ,verwurzelt in der Bevölkerung“ und für viele, gerade die Älteren, ,heilig“ und ,extrem wichtig“ (Interview am 21. Juli 2009). Diese Sakralisierung lässt die neuartige Raum- und Identitätskonstruktion eben nicht als Resultat von gemeinschaftlicher Konsensfindung und touristischer Kommodifizierung erscheinen, sondern als jeder Diskussion und Zirkulation entzogen und nicht (ver-)handelbar. Die Regionalentwicklung des „Kulturerbes“ produziert also religiös-säkulare Hybride. Es wirkt paradox, dass eine Raumkonstruktion bereits da ist, aber erst gemacht werden muss. Bruno Latour nennt solche Gebilde in Anlehnung an den Fetisch-Begriff factish (vgl. Latour, 2010). Das Projekt „Sakrale Landschaft“ bezeichnet der Regionalmanager als mit dem Wachstum ei- nes Baums vergleichbaren ,immerwährenden, sich erweiternden Prozess", der sich in der Vernetzung touristischer Destinationen über administrative Grenzen des Landkreises, des Bundeslands, der Nation hinaus erstrecke, etwa über Pilgerwege bis nach Thüringen oder in die Tschechische Republik. Das natürliche Wachstum ende aber dort, wo die Grenze der Gebietskategorie „ländlicher Raum“ erreicht sei (Interview am 21. Juli 2009).

Die Raumproduktion der ,Sakralen Landschaft“ kann als Governing by Community (Rose, 1999, 2000) begriffen werden - als Regierungstechnik, die über die Region und ihre Geschichte enge Beziehungen zwischen Gemeinschaft, Identität und Subjektivität herstellt und dabei therapeutische Ziele formuliert, denen zufolge „Gemeinschaft“ Objekt subjektiver Identifikation, der Entdeckung eines „eigentlichen Selbst“ sei (Rose, 1999:196). Gemeinschaft ist dabei „natürliches" Residuum von Authentizität und Beziehungsdichte etwas, mit dem sich Subjekte „[...] existentiell, traditionell, emotional und spontan und allem Anschein nach jenseits und vor aller kalkulierten Abschätzung des Eigeninteresses“" identifizieren sollen (Rose, 2000:84) - und zugleich das Produkt eines Regierungs- und Innovationsprozesses, der durch Eigeninitiative und zivilgesellschaftliches Engagement vorangetrieben wird (Rose, 1999, 2000). Gemeinschaft in diesem Sinne kann als weiterer factish bezeichnet werden (vgl. Latour, 2010).

Die EU-Förderung der „Sakralen Landschaft“ als „Inwertsetzung kultureller Besonderheiten und Güter" macht einen Sprachgebrauch erforderlich, der kategoriale Grenzziehungen zwischen Religiösem und Säkularem bestätigt, um in der Praxis beide Bereiche zu Hybriden zu verflechten (vgl. Latour, 1991). Natürlich, so der Regionalmanager, sei die Religion im Konnersreuther Placemaking die treibende Kraft. Aber eben nicht im Förderantrag! Die „saubere Trennung“ von Religion und Kultur müsse gewahrt werden, um den Fördergeber zu überzeugen - „obwohl es dann natürlich keinem verboten ist, diese Struktur zu nutzen, um sein Seelenheil zu finden“ (Interview am 21. Juli 2009). Dieser Gebrauch kategorialer Trennung ist taktisch - ist sich doch der Regionalmanager der eindeutig religiösen Interessen bewusst: Bei Konnersreuth handele es sich schließlich, so betont er, um einen „knallharten Wallfahrtsort“ (ebd.). Religiöse Artikulationen universaler Wahrheitsansprüche wie auch die bekannten Betrugsvorwürfe werden aus dem konsensualen Prozess der Raumproduktion ausgeklammert: Das Regionalmanagement sei „nicht die Clearing-Stelle“; für die „Inwertsetzung kultureller Besonderheiten“ sei einzig relevant, ,diese Struktur anzuerkennen: dass sie da ist" (ebd.).

\section{Sakralisierende Diskurse des Sühneleidens}

Bei meinem ersten Besuch in Konnersreuth anlässlich des zweiten „Großen Gebetstages um die Seligsprechung der Dienerin Gottes Therese Neumann“ im September 2006 
überraschte mich die Stille des Ortes und das fast vollständige Fehlen einer kommerziellen Vermarktung. Die etwa vierhundert TeilnehmerInnen des jährlich stattfindenden Großen Gebetstags - kleinere finden monatlich statt - verließen den Ort nach dem Abschluss einer abendlichen Lichterprozession zum Grab auf dem Gemeindefriedhof recht schnell. Ebenso überrascht war ich, welch große Rolle Themen eines als vorkonziliar geltenden katholischen Devotionalismus (vgl. Orsi, 2010) - Verlöbnis, Fürsprache, Heilung - in meinen Gesprächen mit Besuchern und BewohnerInnen des Orts spielten. Neben dem Grab zieht vor allem das Wohn- und Geburtshaus Therese Neumanns („Reslhaus“), seit April 2005 als Andachtsstätte geöffnet, Pilger aus der Oberpfalz, Bayern, den katholischen Nachbarländern, der europäischen Diaspora, aber auch vereinzelt aus den USA, China, Indien oder Brasilien an. Diese wollen vor allem das „Leidenszimmer“ betrachten, in dem Therese Neumann zwischen 1926 und 1962 in regelmäßigem Rhythmus an der Passion Jesu Christi teilnahm, und ihre „Blutwäsche“, die in einer Vitrine im „Reslhaus“ ausgestellt wird. Der „Blutwäsche“ - der Nachtwäsche Therese Neumanns, die Spuren des getrockneten Bluts aufweist, das während ihres Miterlebens der Passion Christi aus ihren Stigmata quoll, und die von ihren Familienmitgliedern aufbewahrt wurde - werden zahlreiche Heilungen zugeschrieben. Zugleich repräsentiert die „Blutwäsche“ das Sühneopfer Christi - und damit, den kirchlichen Akteuren im Seligsprechungsprozess zufolge, das heilige Sakrament der Eucharistie, das heilige Messopfer. Schließlich sei Therese Neumanns Leiden als aktive Mitwirkung am Erlösungswerk Christi, an der liebenden, freiwilligen Selbsthingabe des Menschensohnes für die Sünden der Welt zu verstehen - im Sinne des Apostels Paulus: ,Jetzt freue ich mich in den Leiden, die ich für euch ertrage. Für den Leib Christi, die Kirche, ergänze ich in meinem irdischen Leben das, was an den Leiden Christi noch fehlt.“ (Kol 1, 24; Wörner, 2015:11). Die Lokalität Konnersreuth, der Körper Therese Neumanns und die Materialität dessen, was durch seine Berührung zur Reliquie wurde, werden damit als Ausgangspunkt einer religiösen Raumproduktion verstanden, die alles Partikulare ins Universale transzendiert: Das katholische Versprechen der Erlösung betrifft die ganze Welt.

Gleichzeitig wird Therese Neumann fest mit einem Autochthonen des „einfachen Volks“ und mit dem Regionalen identifiziert und damit gleichsam ethnifiziert: In seiner Predigt zur Eröffnung des Seligsprechungsprozesses stellt der damalige Bischof Gerhard Ludwig Müller Therese Neumann als typische Oberpfälzerin dar: Therese, so Müller, ,[...] wuchs in einer nüchternen und kraftvollen Frömmigkeit auf und bewahrte sich immer die innere Geradlinigkeit des oberpfälzischen Menschenschlags, der geprägt ist von einer rauen Natur und einer bäuerlichen Kultur, die den Menschen zur Strenge gegen sich selbst erzieht, aber auch zur Unerschütterlichkeit des Glaubens [...]." (ebd.). Die zyklische Wiederholung des Leidens Therese Neumanns wie der Feier des eucharistischen Opfers vermittelt also zwischen der - von Müller in völkischen Begriffen formulierten - regionalen Identifizierung der Gläubigen und der De-Identifizierung ihrer Erlösung.

Der Leiter des Seligsprechungsprozesses, Domvikar Georg Schwager, deutet die Eucharistie als „Erbe“, das in der dynamischen Spannung von Vergangenheit, Gegenwart und Zukunft steht: als das ,,[...] ewige und lebendige, uns vom Herrn im Sakrament seines Leibes und Blutes hinterlassene Erbe, das wir ständig neu durchdenken und vertiefen müssen“ (o. A. [Schwager, G.], 2011:6). Diesem Erbe sollen wir uns, so Schwager, in der heiligen Messe nähern (ebd.) - nicht zuletzt in Konnersreuth, das, so sein Wunsch, ,[...] in den nächsten Jahren und Jahrzehnten zu einem Pilgerort und Glaubenszentrum [... ]“ (o. A. [Schwager, G.], 2005:14) werden solle. Das „Erbe“ der Volksheiligen wird damit als kirchliches „Erbe“ angeeignet - es wird sakralisiert.

Die Predigten der Bischöfe aus dem süddeutschen und österreichischen Raum, die zu den jährlichen Großen Gebetstagen um die Seligsprechung Therese Neumanns nach Konnersreuth eingeladen werden, sind von Vorbehalten gegenüber der säkularen Moderne geprägt. Die Predigten prägt ein apokalyptischer Ton, der die Menschen der Neuzeit zur religiösen Umkehr mahnt. Nur religiöse Handlungen - Beten, Sühne und Leiden - seien imstande, die Rettung der Welt zu erwirken. Die wichtigste Rolle komme dabei religiösen Experten zu: Die wahre Erneuerung, heißt es immer wieder, gehe vom Altar aus - vom Priester, dessen wichtige Aufgabe das Opfer sei (Müller, 2010:30). Die Prediger auf den Gebetstagen empfehlen das religiöse Opfer der Eucharistie als rituelles Mittel zur Lösung einer kosmologischen Krise, zur Wiederherstellung einer durch die Kräfte der Moderne gestörten universalen göttlichen Ordnung (vgl. Henaff, 2009:241-311) - eine antipolitische „Archi-Politik“ (Rancière, 2002:75ff.). Neben einer häufigen Teilnahme an der Messfeier empfehlen die Prediger Praktiken der ewigen Anbetung, Sühneleiden, Gebete und eine „Umkehr der Herzen" (Konnersreuther Nachrichten 63/2005) - religiöse Formen der Subjektivierung, um das Heil der Welt zu erlangen. Säkulare politische Auseinandersetzungen um das Gemeinwohl dagegen werden verworfen: „Unsere Strahlkraft wäre wohl stärker, wenn wir - statt endloser Diskussionen, Konferenzen, Strukturdebatten, Sitzungen und dergleichen mehr - uns deutlicher als betendes, anbetendes Volk Gottes erwiesen.“ (ebd.) Mit ihrem Verständnis des Messopfers als aktives Mitwirken am Erlösungswerk Christi wenden sich die Priester auf den Gebetstagen nicht nur gegen ein Verständnis der Eucharistie als reine Erinnerungshandlung und „Gemeinschaftsmahl“", sondern auch gegen religiöse und religionsähnliche Formen individualistischer Heilssuche und Selbstperfektion. Gegen den Glauben der Moderne an die Möglichkeit der diesseitigen Überwindung menschlichen Leids und gegen das Primat der Selbstverwirklichung der gegenwärtigen „Wellness-Religion“ (ebd.) empfehlen die Bischöfe Therese Neumann als Vorbild demütiger Entsagung: „Ihr Leitspruch lautete: ,Mir ist alles recht, Gesundheit und Krank- 
sein, Leben und Sterben. [...] Ich will keinen anderen Willen haben als den des Heilands. ““ (Müller, 2005). Auf dem vierten Großen Gebetstag 2008 erteilt der Passauer Bischof Wilhelm Schraml dem „Leidensverbot“ der heutigen Gesellschaft, in der ,[...] der Mensch nicht für einen Kreuzweg, sondern für Glück und Genuss programmiert scheint [...]“ (Schraml, 2009:16), sowie der Perversion der „Kreuzesreligion“"zur „Wohlstandsreligion“ (Schraml, 2009:17) eine Absage. Erlösung sei nur durch das Leid, nicht vom Leid zu haben (ebd.). Der Trost des Gekreuzigten sei ,[... ] der einzige Trost, der nicht vertröstet.“ (ebd.: 17f.)

In der kategorischen Trennung profaner und sakraler, säkularer und religiöser Sphären, welche die kirchlichen Amtsträger auf den Gebetstagen befürworten, drückt sich eine brüske Ablehnung der säkularen Moderne und ein dezidierter Rückzug religiösen Handelns auf den kirchlichen Bereich der Liturgie aus. Ihre Ablehnung einer „Wohlstandsreligion“ und ihr Beharren auf katholischen Wahrheitsansprüchen impliziert eine Absage an „postsäkulare“ Instrumentalisierungen von Religion als touristischer Ware, individuellem Therapeutikum oder - wie von Habermas und Casanova vorgestellt - moralischer Ressource in der Selbstverständigung einer pluralistischen Zivilgesellschaft.

\section{Reslgarten: Ort des kreativen Milieus und der Heilung}

Während der kirchliche Diskurs Therese Neumann als Sühneopfer deutet, das im partikularen Ort Konnersreuth an der Verwirklichung universalen Heils mitwirkt, rückt die Raumproduktion zivilgesellschaftlicher AkteurInnen im Reslgarten die selbstbezügliche Realisierung eines ,authentischen“ Selbst durch Wellness, Heilung und Konversion ins Zentrum. Die 2006 abgeschlossene Rekonstruktion des historischen Gartens, in dem Therese Neumann Blumen für die Kirchenaltäre züchtete und sich vom Publikumsbetrieb erholte, war das erste LEADER-Projekt in Konnersreuth. Von zwei weiblichen Vorstandsmitgliedern des Obst- und Gartenbauvereins (OGV) initiiert, beteiligten sich daran 45 ehrenamtliche HelferInnen aus Konnersreuther Vereinen, Pfarrgemeinde und Marktrat, deren Eigenleistung auf 3400 Stunden unbezahlter Arbeit im Wert von 60000 Euro beziffert wurde. LEADER und - ein Novum - die Bischöfliche Finanzkammer Regensburg förderten das Projekt mit je 70000 Euro. Etwa dreißig meist weibliche ehrenamtliche Mitarbeiter teilen sich in die Pflege des Gartens und die Betreuung seiner BesucherInnen. Ein Steingarten mit Hochbeeten, ein kleiner Teich, Blumenbeete, Gewächshaus, Obstbaumwiese, Gartenhäuschen und ein Schuppen mit originalen Arbeitsgeräten Therese Neumanns zählen zum Inventar des Gartens - eine Art Freilichtmuseum, das weniger an die Stigmatisation als an die historische Gartenkultur der 1950er Jahre erinnert und die „Gärtnerin“ Therese Neumann als typische Oberpfälzer Landfrau zeigt. Einen einladenden Raum eines kleinen Neubaus, der der Bewirtung und Information der BesucherInnen dient, haben sich die Frauen als offene Sphäre zum nachbarschaftlichen Austausch angeeignet. Im Jahr der Eröffnung wurde der „Reslgarten“ in die grenzübergreifende Landesgartenschau Cheb - Marktredwitz integriert. 10000 Neugierige besuchten den Garten. ${ }^{4}$ Diese Integration verknüpfte die Verehrung Therese Neumanns mit den Themen Tourismus, Gartenbau und Landschaftsarchitektur und mit den Zielen des Regionalmarketing.

In der Selbstdarstellung ihrer Raumproduktion verknüpfen die AkteurInnen Diskurse der Regionalentwicklung und der Heiligenverehrung. Das Regionalmanagement sieht im Reslgarten die Keimzelle eines „kreativen Milieus“ und lobt das selbständige unternehmerische Handeln der Vereinsvorsitzenden. ${ }^{5}$ Diese setzt sich öffentlich für eine verstärkte private bzw. privatwirtschaftliche Eigeninitiative bei der touristischen Vermarktung des Konnersreuther „Alleinstellungsmerkmals" Therese Neumann ein und stilisierte die Volksheilige zum Vorbild kreativen Unternehmertums: Thereses Initiative, ihrem Durchhaltevermögen und Verhandlungsgeschick sei die Errichtung der beiden Klöster des Orts zu verdanken. ${ }^{6}$ Die Entdeckung, dass Therese Neumann im Jahr 1928 Gründungsmitglied des Obst- und Gartenbauvereins war (das einzig weibliche zudem), ${ }^{7}$ ermöglichte es den Frauen, sich die Volksheilige auf neue Weise als Patronin ihres Vereins und Vorreiterin ihres zivilgesellschaftlichen Engagements anzueignen. Therese Neumann selbst, so die Vereinsvorsitzende bei der Einweihungsfeier des Gartens, habe bei der Rekonstruktion des Gartens „von oben herab“ mitgeholfen und dazu beigetragen, dass ,unüberbrückbare Probleme plötzlich gelöst waren“. 8

Transzendenzbezüge der Heiligenverehrung durchkreuzen immer wieder die säkulare Raumkonstruktion des Gartens als Ort der Ruhe und Erholung. Die Raumkonstruktion des Gartens im Rahmen der touristischen „Sakralen Landschaft" regionalisiert die "Natur“ des Gartens und stellt die Bepflanzung der Hochbeete als Repräsentation der botanischen Vielfalt der Oberpfalz en miniature dar. Der naturkundliche Aspekt wird durch kleine Tafeln mit lateinischen Klassifikationen und entsprechenden deutschen Bezeichnungen der Pflanzen hervorgehoben. Therese Neumann, so die Ehrenamtlichen, sei mit der regionalen Natur vertraut gewesen und habe - als Naturheilerin - Kräuter der heimischen Flora gepflückt. Die Raumkonstruktion des Regionalen verknüpfen die Ehrenamtlichen mit Themen ökologischer Nachhaltigkeit und dem hagiographischen Motiv der "Liebe zur Schöpfung“ Therese Neumanns.

Zugleich laden die Ehrenamtlichen das Ortstypische des Gartens mit religionsähnlichen Sinnkonstruktionen des mo-

\footnotetext{
${ }^{4}$ Neuer Tag (Weiden/Opf.) vom 14. Juni 2006.

${ }^{5}$ Interview vom 21. Juli 2009.

${ }^{6}$ Wortmeldung auf der Bürgerversammlung vom 24. Juli 2009.

${ }^{7}$ Gespräch am 13. Juli 2009.

${ }^{8}$ Neuer Tag (Weiden/Opf.) vom 16. Mai 2006.
} 
dernen Wellness-Tourismus auf. Sie beschreiben den etwas abseits des Ortskerns gelegenen Reslgarten, der einen weiten Blick über Wiesen und Ebenen bis zur nächsten Stadt erlaubt, als Ort des Wohlbefindens und spirituellen Kraftort. Kleine Tontafeln in den Beeten mit Sinnsprüchen aus globalisierten spirituellen Traditionen, etwa von Khalil Gibran, verstärken diese Raumkonstruktion. Im Reslgarten, vertraut mir eine Mitarbeiterin an, vergesse sie oft die Zeit, ihre Krankheit (,Keiner von uns hier ist richtig gesund“), die Sorgen des Alltags, und sei ganz im Hier und Jetzt. ${ }^{9}$ Die Raumkonstruktion eines „,Orts der Fülle“, an dem ,[... ] das Leben voller, reicher, tiefer, lohnender, bewundernswerter und in höherem Maße das [ist], was es sein sollte“ (Taylor, 2009:18), ermöglicht die Vermittlung säkularer und religiöser Bedeutungen.

Die Auseinandersetzung mit chronischer bzw. unheilbarer Krankheit, mit Sterben und Tod sowie mit Heilungserfahrungen - mit unvermittelten biographischen Brüchen, welche Menschen vollkommen verändern und neue Subjektivierungen ermöglichen - spielen in den Erzählungen der MitarbeiterInnen im Garten immer wieder eine Rolle. Eine Ehrenamtliche stellt ihre Arbeit als „Verlöbnis“ Therese Neumanns dar. Während der Arbeiten an der Rekonstruktion sei sie lebensgefährlich erkrankt. Ihr Versprechen, den Garten im Falle ihrer Heilung als Dankesgabe fertigzustellen, habe schließlich zur Genesung geführt. Ihr gegenwärtiges Wohlbefinden deutet sie als Resultat eines anhaltenden Bewusstseinswandels nach der Heilung: Seitdem empfinde sie die Schönheit der Welt stärker als je zuvor und widme ihre ganze Tatkraft der Ehre Therese Neumanns. ${ }^{10}$

Eine andere Mitarbeiterin stellt den Garten als Endpunkt einer spirituellen Suchbewegung für die BesucherInnen dar. Damit verknüpft sie ein für die Religiosität im gegenwärtigen ,Zeitalter der Authentizität" paradigmatisches Thema das Streben nach Selbstverwirklichung (vgl. Taylor, 2009) mit der Formulierung eines religiösen Ziels. Immer wieder kämen „Suchende“ in den Garten, die etwas auf dem Herzen hätten. In selbstläufigen Gesprächen kämen sie häufig über Probleme in Beruf oder Partnerschaft auf den Sinn des Lebens und den Glauben zu sprechen. Einige Suchende habe sie bereits zur Taufe bewegen können. Bei ihrer Missionstätigkeit hole sie des öfteren den Rat des Ortspfarrers ein, etwa angesichts ihrer Bekehrung eines homosexuellen Paares ein heikles Thema für die katholische Kirche. ${ }^{11}$ Ihre Begegnung mit „Suchenden“ - eine Begegnung, deren Potentialität und Offenheit sie hervorhebt - wird damit im institutionellen Rahmen der Amtskirche eingehegt. Es ist offensichtlich, dass sich die Mitarbeiterin nach ihrem Vorbild Therese Neumann stilisiert, die ihre Missionstätigkeit ebenfalls unter der seelsorgerischen Obhut ihres Beichtvaters, Pfarrer Joseph Naber, ausübte (Steiner, 1985). Die plurale Raumkonstruktion des Gartens ermöglicht es ihr, BesucherInnen flexibel

\footnotetext{
${ }^{9}$ Gespräch am 20. Juli 2009 .

${ }^{10}$ Gespräch am 22. November 2007.

${ }^{11}$ Gespräch am 22. November 2007.
}

als VerehrerInnen Therese Neumanns, Naturfreunde oder als Menschen auf einer Suche nach dem Sinn des Lebens anzusprechen und damit situationsbedingt zwischen touristischer Dienstleistung und spiritueller Sorgearbeit zu wechseln.

Die Tourismusbroschüre der Gemeinde, an deren Erstellung Ehrenamtliche des Reslgartens maßgeblich beteiligt waren, vermischt Semantiken der Wellness und des Religiösen auf spielerische Weise und trägt damit zur Verunsicherung von postsäkularen Vorannahmen über die vermeintliche naive Unmittelbarkeit des religiösen Glaubens der touristisch besuchten Anderen (Žižek) bei. Sie bewirbt unter dem Claim „Konnersreuth. Was Leib und Seele gut tut“ das besondere Angebot, „Glauben hautnah [zu] erleben“ (Markt Konnersreuth, 2008). Die Broschüre ziert ein Sinnbild, welches eine Mitarbeiterin auf meine Nachfrage als Darstellung einer Mutter, die ihr Kind im Arm wiegt, und/oder einer Hostie über einem Messkelch beschreibt. ${ }^{12}$ Durch diese Mehrdeutigkeit wird die Raumkonstruktion der Wellness mit der eucharistischen Verehrung Therese Neumanns verknüpft, religiöse und säkulare Konzeption werden im Rahmen der Regionalentwicklung der „Gesundheitsregion“ auf den gemeinsamen Nenner der Geborgenheit und Fülle gebracht. Die Raumkonstruktion spielt mit einer postsäkularen „Dispersion des Religiösen“, einer Aneignung religiös konnotierter Ästhetiken und Semantiken für nicht-religiöse Zwecke im zeitgenössischen Format der Wellness, die ,[...] eine gewisse Aura oder Assoziativkraft, in Richtung Religion“ hat (Höhn, 2011:84) - und kann als (Wieder-)Aneignung dieser Ästhetiken für religiöse Zwecke verstanden werden.

\section{Projekt Schafferhof: Konfliktpotentiale im postsäkularen Placemaking}

Im Projekt „Schafferhof“, dem Ausbau eines leerstehenden Bauernhofs im Ortskern zum „Dokumentations- und Begegnungszentrum“ unter der Trägerschaft der Kommune Konnersreuth, zeigt sich die Konflikthaltigkeit des postsäkularen Placemaking. Im Zentrum des Großprojekts steht die Einrichtung eines „Reslmuseums“, welches Therese Neumann einer breiten Öffentlichkeit bekanntmachen soll. Die Aufnahme des Hofs in die Denkmalschutzliste stellte eine strategische Maßnahme dar, um Fördergelder einwerben zu können. Ein rein religiöses Projekt, etwa ein Pilgerzentrum, hieß es, werde von der EU nicht gefördert.

Nachdem der Unmut der KonnersreutherInnen über eine vermeintliche Geheimhaltungspolitik des Marktrats immer lauter geworden war, wurde auf einer Bürgerversammlung im Juli 2009 die Entstehungsgeschichte des Projekts offengelegt und zugleich das drohende Scheitern des Projekts bekanntgegeben. Die Projektkosten beliefen sich auf 6,7 Millionen Euro; Fördergelder in Höhe von etwa 5 Millionen Euro, unter anderem aus dem Städtebauprogramm des Bundes, EU-Programmen sowie des Denkmalschutzes, standen be-

\footnotetext{
${ }^{12}$ Gespräch am 9. August 2008.
} 
reit. Den Eigenanteil von rund 1,5 Millionen Euro konnte die Gemeinde aufgrund der schlechten Haushaltslage nicht aufbringen. ${ }^{13}$ In der öffentlichen Debatte wurden Bruchlinien zwischen lokalen Interessengruppen und damit die Brüchigkeit von Projekten des Governing by Community deutlich, die auf die Partizipation der Subjekte und die Herstellung von Konsens angewiesen sind. Während Bürgermeister, Markträte, der Pfarrer und Vertreter des „kreativen Milieus“ zum einmütigen Handeln aufforderten und die Untrennbarkeit von Kirchen- und Marktgemeinde betonten, kritisierten einige BürgerInnen diese Rhetorik einmütiger „Gemeinschaft“. Weder sei die Nachhaltigkeit und Profitabilität des Projekts für die Gemeinde noch die Verteilung eventueller Gewinne offengelegt worden. BürgerInnen klagten lautstark eine stärkere finanzielle Beteiligung der „Kirche“ ein, die schließlich genauso vom Projekt profitiere wie die politische Gemeinde: Das Bistum Regensburg weigere sich, Förderzusagen zu geben. Skandalisiert wurden auch demokratische Defizite und die Intransparenz von Planungs- und Entscheidungsprozessen. Zudem weigerten sich lokale Vereine, dem Imperativ der "Gemeinschaft" zu folgen, und erteilten der Aufforderung der politischen Marktgemeinde, Vorstellungen und Ansprüche bezüglich der Nutzung des Hofs anzumelden, eine Absage: Sie fürchteten den Autonomieverlust zivilgesellschaftlicher Strukturen und den Zugriff auf ihre finanziellen Ressourcen. ${ }^{14}$ Damit musste der „breite Rückhalt“ des Projekts in der „Bevölkerung“ gefährdet erscheinen.

Vor allem aber die Strategie der Prädikatierung und Inwertsetzung von „Kulturerbe“ und der Versuch der Identifizierung von Kulturerbe und "Gemeinschaft" waren nur schwer zu vermitteln. In einem Powerpoint-Vortrag erläuterte der federführende Architekt den Anwesenden die Siedlungsgeschichte des Orts, die Baugeschichte des Ensembles sowie die geplante Rekonstruktion. Ausführlich begründete er den Denkmalschutz des Ensembles, seinen „ortsbildprägenden Charakter" und seine regionaltypische Bauweise. Seine „bewusstseinsbildenden Maßnahmen“ überzeugten nur bedingt: Während einige Anwesende begrüßten, dass die kunsthistorische Betrachtung des Hofs ihre Blickweise verändert habe, sprachen Vertreter der älteren Generation dem Bauwerk weiterhin jegliche Fähigkeit ab, Gemeinschaft und geschichtlich gewachsene Identität Konnersreuths zu repräsentieren. ${ }^{15}$ Sie, mit dem bäuerlichen Leben seit Kindheit vertraut, betrachteten die verfallenden Gemäuer nicht hinsichtlich ihres Ausstellungswerts, sondern ihres Gebrauchswerts: Mit der Modernisierung der Landwirtschaft in den 1970er Jahren war der Hof zu eng, ein Umzug des Betriebs notwendig und das Gebäudeensemble wertlos geworden.

Nach dem Ende der Versammlung begründeten einige, die die 1962 verstorbene Therese Neumann noch persönlich gekannt hatten, ihre Ablehnung des Großprojekts in privaten

\footnotetext{
${ }^{13}$ Bürgerversammlung am 24. Juli 2009.

${ }^{14}$ Wortmeldungen auf der Bürgerversammlung am 24. Juli 2009. ${ }^{15}$ Ebd.
}

Gesprächen mit dem „Willen der Resl““: Diese habe ein touristisches Spektakel nicht gewollt; sie - die Stigmatisierte, über die die internationale Presse berichtete - sei im übrigen ein „Mensch wie du und ich“ gewesen. Dass Therese Neumann mit dem „Reslwetter“ - plötzlichen Regenschauern, Gewittern, Schneestürmen und Wetterwechseln an ihren Gebetstagen - immer wieder dafür sorge, dass „,die Fremden“ fernblieben, führen sie als Beweis an, dass die Volksheilige aktiv an der Raumproduktion der ",Sakralen Landschaft" teilnimmt: ${ }^{16}$ indem sie diese nämlich stört und damit die touristischen wie amtskirchlichen Identifizierungen regionaler Gemeinschaft unterbricht. Dass diese Argumentation mit dem Eigensinn der „Resl“ keinen Eingang in die öffentliche Debatte fand, kann als Ausdruck einer ,moralischen Sensibilität" (Habermas) für die Notwendigkeit verstanden werden, die Grenzen zwischen säkularer Öffentlichkeit und privater Religiosität aufrechtzuerhalten. Nachdem der Marktrat im Jahr 2012 die Realisierung des Projekts Schafferhof bis 2016 ausgesetzt hatte und die zugesagten Fördergelder verfallen waren, sprach sich auf einem „Bürgerentscheid“ im Oktober 2014 eine klare Mehrheit für einen Abriss des historischen Hofs und einen anschließenden Neubau aus. Der Antrag des Marktrats an die Denkmalschutzbehörde um Erlaubnis, das Baudenkmal abreißen zu dürfen, wurde jedoch abgelehnt; das Vorhaben der Marktgemeinde, gegen diesen Beschluss zu klagen, wurde wegen mangelnder Erfolgsaussichten verworfen. ${ }^{17}$ Im März 2017 reichte der Marktrat einen erneuten Antrag auf Städtebauförderung für die Sanierung des Schafferhofs ein. ${ }^{18}$

\section{Resümee}

Das postsäkulare Placemaking des Wallfahrts- und Urlaubsorts Konnersreuth lässt sich als potentiell konflikthaftes Nebeneinander verschiedener situationsabhängiger Grenzziehungen zwischen dem Säkularen und Religiösen beschreiben, die in Wertzuschreibungen des materiellphysischen und spirituellen „Erbes“ Therese Neumanns und ihrer Heimatgemeinde Konnersreuth vorgenommen werden. Im Konnersreuther Placemaking werden Verknüpfungen des Säkularen und Religiösen, lokaler und potentiell globaler Prozesse - der kirchlichen Seligsprechung und der touristischen Inwertsetzung von regionaler Besonderheit und kulturellem Erbe - sowie von Deutungen der Vergangenheit und Zukunftsentwürfen hergestellt, die teilweise stark umstritten sind (Massey, 1994, 1995). Mein Artikel versucht dabei verschiedene Positionen zu unterscheiden: Während das Regionalmanagement die Inwertsetzung der „Sakralen Landschaft“ als Ressource touristischer Ökonomie und regionaler Identität betreibt und Religiöses als regionale Besonderheit einer globalen

\footnotetext{
${ }^{16}$ Gespräche am 24. Juli 2009.

${ }^{17}$ Neuer Tag vom 14. Mai 2016.

${ }^{18}$ Neuer Tag vom 16. Mai 2017.
} 
Kulturökonomie einverleibt, beharren Akteure des kirchlichen Seligsprechungsprozesses auf den säkular/religiösen Grenzziehungen der säkularen Moderne und verknüpfen im Diskurs um das Sühneleiden Therese Neumanns das Lokale und Regionale mit der Verheißung der Transzendenz alles Fragmentarisch-Besonderen. Das Nebeneinander der Promotion eines vorkonziliar wirkenden Heiligenkults und der EU-Entwicklung einer strukturschwachen Grenzregion mittels Inwertsetzung von Kulturerbe wird ermöglicht durch die Vieldeutigkeit materieller Kultur und die Multitemporalität, die laut Doreen Massey places of the past kennzeichnet (Massey, 1995). Eine weitere Position lässt sich im WellnessAngebot des „Reslgartens“ feststellen, das im Rahmen der "Gesundheitsregion" Tirschenreuth Themen der Heilung und Bekehrung verknüpft und damit an einer postsäkularen Vermischung des Religiösen und Säkularen arbeitet, die der amtskirchlichen Trennung dieser Sphären widerspricht. Die Raumkonstruktion des „Reslgartens“ kann als religiöse Wiederaneignung religiöser Semantiken nicht-religiöser Formate begriffen werden (vgl. Höhn, 2011), bei der die Grenzen zwischen Religiösem und Säkularem, zwischen der regionalen Identifizierung von Ort und Gemeinschaft und der universalen De-Identifizierung der Gnade (vgl. Badiou, 2002) verschwimmen. Die religiöse Aneignung touristischer Formate des Kulturerbes und der Wellness und die damit verknüpfte Herausforderung der postsäkularen Kulturalisierung des Religiösen (Žižek) geschieht eher tongue in cheek - taktisch, spielerisch und subversiv - als konfrontativ oder fundamental.

Eine weitere Position lässt sich in privaten Äußerungen älterer Zeitgenossen Therese Neumanns identifizieren. Diese berufen sich auf den Willen der Volksheiligen, um eine touristische Inwertsetzung abzulehnen sowie den amtskirchlichen Sakralisierungen Grenzen zu setzen. Das Placemaking in Konnersreuth erscheint damit als postsäkularer Prozess, in dem das eigenwillige Beharren auf der Fähigkeit der Volksheiligen, unberechenbar und manchmal jähzornig ins Geschehen einzugreifen, den Eigensinn der „Bevölkerung "legitimiert, religiösen wie gouvernementalen Anrufungen gleichermaßen das Gehör zu verweigern. Für die Pilgernden, die nach Konnersreuth kommen, um von Therese Neumann Heilung von Krankheiten, Schutz vor Unfällen, Beistand in familiären oder finanziellen Angelegenheiten zu erbitten, liegt die „Besonderheit“ des Orts in der Gewissheit begründet, dass hier jedermann Heilung finden könne. Gnade, so Alain Badiou in seiner Interpretation des Apostels Paulus, eröffnet eine singuläre Wahrheit, die ,[...] allen angeboten oder für jeden bestimmt [ist], ohne dass irgendeine vorausgesetzte Zugehörigkeit dieses Angebot oder diese Bestimmung einschränken könnte“ (Badiou, 2002:29). Diese Gnade entzieht sich aber - unberechenbar und ungewiss - jedem kommunitären Zugriff, ,„[... ] mag es sich um ein Volk, eine Stadt, ein Reich, ein Territorium oder eine soziale Klasse handeln." (Badiou, 2002:13) Die Äußerungen der übernatürlichen Agency Therese Neumanns - plötzlicher Regen,
Schneefall und Hagelschauer, aber auch die Erde des Grabs und die Reliquien -, spielen, darin sind sich viele einig, eine wichtige Rolle im Placemaking (Orsi, 2010). Diese ÄuBerungen unterbrechen zumindest zeitweise die identifizierende Logik des Governing by Community, die reibungslose Verwandlung der Hinterlassenschaften Therese Neumanns in das universelle touristische Format „Kulturerbe“, aber auch die autochthone Identifizierung von Kirche und „einfachem Volk“. Das Postsäkulare besteht im Fall Konnersreuth im Beharren auf einer übernatürlichen Präsenz, die sich der Verfügung und Verzweckung eigensinnig entzieht.

Datenverfügbarkeit. Aufgrund der Vertraulichkeit mancher Informationen wurden die von mir geführten Interviews nicht veröffentlicht und meine GesprächspartnerInnen anonymisiert.

Interessenkonflikt. Der Autor erklärt, dass kein Interessenkonflikt besteht.

Danksagung. Einen ersten Entwurf meiner Argumentation habe ich auf der Interdisziplinären Jahrestagung des Zentralinstituts „Anthropologie der Religion(en)“ der Friedrich-AlexanderUniversität Erlangen-Nürnberg in Kooperation mit dem AK Religionsgeographie im Juni 2015 vorstellen können. Ich danke den OrganisatorInnen der Tagung für diese Gelegenheit und den TeilnehmerInnen für ihre Kritik und Anregungen. Mein besonderer Dank gilt dem Editor der Geographica Helvetica, Benedikt Korf, sowie den beiden anonymen GutachterInnen meines Beitrags für ihre hilfreichen Kommentare und Anmerkungen. Vor allem aber möchte ich mich bei meinen GesprächspartnerInnen in Konnersreuth und der Region Nördliche Oberpfalz herzlich bedanken.

Edited by: Benedikt Korf

Reviewed by: two anonymous referees

\section{Literatur}

Asad, T.: Formations of the Secular. Christianity, Islam, Modernity, Stanford, Stanford University Press, 2003.

Badiou, A.: Paulus. Die Begründung des Universalismus, München, sequenzia, 2002.

BStMLF: Bayerisches Staatsministerium für Landwirtschaft und Forsten, Gemeinschaftsinitiative LEADER+ im Freistaat Bayern, Programm 2000-2006, Entscheidung der Kommission Nr. K (2001) 4324 vom 17. Dezember 2001.

Beaumont, J. and Baker, Ch.: Introduction. The Rise of the Postsecular City, in: Postsecular Cities. Space, Theory and Practice, Herausgeber: Dies., London, Continuum, 1-14, 2011.

Casanova, J.: Public Religions in the Modern World, Chicago, Chicago University Press, 1994.

Casanova, J.: Europas Angst vor der Religion, Berlin, Berlin University Press, 2009.

Casanova, J.: Erkundungen des Postsäkularen. Rolle und Bedeutung der Religion in Europa, Westend, 8, 68-79, 2011. 
Cloke, P. and Beaumont, J.: Geographies of postsecular rapprochement in the city, Prog. Hum. Geog., 37, 27-51, 2012.

European Commission: Factsheet. The Leader Approach. A Basic Guide. Luxembourg, Office for Official $\mathrm{Pu}-$ blications of the European Communities, 2006, online abrufbar: http://enrd.ec.europa.eu/enrd-static/fms/pdf/ 2B953E0A-9045-2198-8B09-ED2F3D2CCED3.pdf, letzter Zugriff: 12. Juli 2017.

EU-Comm. 2000/C 139/05: Mitteilung der Kommission an die Mitgliedsstaaten vom 14. April 2000 über die Leitlinien für die Gemeinschaftsinitiative für die Entwicklung des ländlichen Raums (Leader+), online aufrufbar: http: //eur-lex.europa.eu/legal-content/DE/TXT/PDF/?uri=CELEX: 32000Y0518(01)\&from=DE, letzter Zugriff: 8. Mai 2017.

Gökarıksel, B. and Secor, A.: Post-secular geographies and the problem of pluralism: Religion and everyday life in Istanbul, Turkey, Political Geography, 46, 21-30, 2015.

Heeg, S.: Politische Regulation des Raums. Metropolen - Regionen - Nationalstaat, Berlin, Sigma, 2001.

Henaff, M.: Der Preis der Wahrheit. Gabe, Geld und Philosophie, Frankfurt am Main, Suhrkamp, 2009.

Höhn, H.-J.: Postsäkulare Moderne? Beobachtungen zur Dispersion religiöser Traditionen, Westend, 8, 80-89, 2011.

Johler, R.: Europäische Orte. Territorialisierungsprozesse im neuen Europa, in: Ort. Arbeit. Körper. Ethnografie Europäischer Modernen, Herausgeber: Binder, B., Göttsch, S., Kaschuba, W., und Vanja, K., Münster, Waxmann, 33-44, 2005.

Kane, P.: "She Offered Herself up": The Victim Soul and Victim Spirituality in Catholicism, Church Hist., 71, 80-119, 2002.

Kirshenblatt-Gimblett, B.: Intangible Heritage as Metacultural Production, Museum International, 56, 52-65, 2004.

LAG InitiAKTIVkreis Tirschenreuth: Regionales Entwicklungskonzept 2000-2006, Tirschenreuth, 2000.

LAG InitiAKTIVkreis Tirschenreuth: Regionales Entwicklungskonzept 2007-2013, Tirschenreuth, online aufrufbar: http://www.initiaktivkreis.de/fileadmin/user_upload/downloads/ REK_2007-2013.pdf (letzter Zugriff: 8. Mai 2017), 2007.

LAG InitiAKTIVkreis Tirschenreuth: Lokale Entwicklungsstrategie 2014-2020, Tirschenreuth, online aufrufbar: http://www. initiaktivkreis.de/fileadmin/user_upload/Leader20142020/LES_ Hauptteil_FINAL_ge\%C3\%A4ndert052015.pdf (letzter Zugriff: 8. Mai 2017), 2014.

Landwehr, A.: Historische Diskursanalyse, Frankfurt am Main, Campus, 2008.

Latour, B.: Wir sind nie modern gewesen. Versuch einer symmetrischen Anthropologie, Frankfurt am Main, Suhrkamp, 1991.

Latour, B.: On the Modern Cult of the Factish Gods, Durham, Duke University Press, 2010.

Low, S. M. and Lawrence-Zuñiga, D.: The Anthropology of Space and Place, Locating Culture, Malden, MA, Blackwell, 1999.

Macdonald, S.: Memorylands. Heritage and Identity in Europe today, London, Routledge, 2013.

Malik, J.: Mobilisierung von Religion in Europa, Frankfurt am Main, Lang, 2010.

Markt Konnersreuth: Konnersreuth. Was Leib und Seele gut tut, Tourismusbroschüre, Konnersreuth, o.J., 2008.

Massey, D.: A Global Sense of Place, Space, Place and Gender, Herausgeber: Dies., Cambridge, Polity Press, 146-156, 1994.
Massey, D.: Places and Their Pasts, Hist. Workshop, 39, 182-192, 1995.

MetroZones (Hrsg.): Urban Prayers. Neue religiöse Bewegungen in der globalen Stadt, Berlin, Assoziation A, 2011.

Meyer, B.: Mediation and the Genesis of Presence. Towards a Material Approach to Religion, Religion and Society, Advances in Research, 5, 205-254, 2014.

Miller, J.: Kultur schafft Werte - weiche Standortfaktoren im ländlichen Raum, in: Landkultur als Standortfaktor, Berichte, Herausgeber: Bayerisches Staatsministerium für Landwirtschaft und Forsten, München, Heft 82/2004, 5-14, 2004.

Molendijk, A. L., Beaumont, J., and Jedan, C.: Preface, in: Exploring the Postsecular. The Religious, the Political, and the Urban, Herausgeber: Dies., Leiden, Brill, ix-xii, 2010.

Müller, G. L. (Bischof von Regensburg): Predigt zur Eröffnung des Seligsprechungsprozesses, online aufrufbar: http://www.therese-neumann.de/seligsprechungsprozess/ predigt-des-bischofs.html (letzter Zugriff: 8. Mai 2017), 13. Februar 2005.

Müller, G. L.: „Der Priester ist ein Mann der Vertrautheit mit Gott““, Therese Neumann Brief, 19, 28-31, September 2010.

Nilsson, M.: Post-Secular Tourism: A Study of Pilgrimages to Santiago de Compostela, Karlstad University Studies, Karlstad, online aufrufbar: http://kau.diva-portal.org/smash/get/diva2:886256/ FULLTEXT01.pdf (letzter Zugriff: 8. Mai 2017), 2016.

o. A. [Schwager, G.]: „Liebe Leser!“, Therese Neumann Brief, 14, 8-14, September 2005.

o. A. [Schwager, G.]: „Das kostbare Geschenk des Glaubens“, Therese Neumann Brief, 19, 5-13, hier: S. 6, September 2010.

o. A. [Schwager, G.]: „Erneuerung der Kirche durch Buße, Eucharistie und Nächstenliebe“, Therese Neumann Brief, 20, 5-14, September 2011.

Oosterbaan, M.: Public religion and urban space in Europe, Soc. Cult. Geogr., 15, 591-602, 2014.

Orsi, R. A.: Introduction to the Third Edition, in: The Madonna of 115th Street, Faith and Community in Italian Harlem, 1880 1950, 3rd Edition, New Haven/London, Yale University Press, ix-xxvi, 2010

Panzer, A.: Licht von drüben. Ein Journalist begleitet das mystische Leben der Therese Neumann, Amberg, Druckhaus Oberpfalz, 1991.

Rancière, J.: Das Unvernehmen. Politik und Philosophie, Frankfurt am Main, Suhrkamp, 2002.

Rose, N.: Powers of Freedom. Reframing Political Thought, Cambridge, Cambridge University Press, 1999.

Rose, N.: Tod des Sozialen? Eine Neubestimmung der Grenzen des Regierens, in: Gouvernementalität der Gegenwart, Studien zur Ökonomisierung des Sozialen, Herausgeber: Bröckling, U., Krasmann, S., Lemke, T., Frankfurt am Main, Suhrkamp, 72109, 2000.

Schraml, W.: „Auftrag der ,Resl': Seelen gewinnen und sie dem Heiland näher bringen“, Therese Neumann Brief, 18, 15-22, September 2009.

Shore, C.: Building Europe, The Politics of European Integration, London, Routledge, 2000.

Steiner, J.: Theres Neumann von Konnersreuth. Ein Lebensbild nach authentischen Berichten, Tagebüchern und Dokumenten, Zürich, Schnell \& Steiner, 1985.

Tauschek, M.: Kulturerbe. Eine Einführung, Berlin, Reimer, 2013. 
Taylor, A.: Ein säkulares Zeitalter, Frankfurt am Main, Suhrkamp, 2009.

Tschofen, B.: Antreten, ablehnen, verwalten? Was der HeritageBoom den Kulturwissenschaften aufträgt, in: Prädikat „Heritage“. Wertschöpfungen aus kulturellen Ressourcen, Herausgeber: Hemme, D., Tauschek, M., und Bendix, R., Berlin, LIT Verlag, 19-32, 2007.

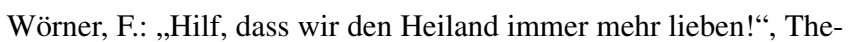
rese Neumann Brief, 24, 11-16, September 2015.

Žižek, S.: Die gnadenlose Liebe, Frankfurt am Main, Suhrkamp, 2001.

Žižek, S.: Die Puppe und der Zwerg, Das Christentum zwischen Perversion und Subversion, Frankfurt am Main, Suhrkamp, 2003. 